\section{P-143 TOO LATE TO INTERVENE: A REVIEW OF THE TIMELINESS OF REFERRALS FOR ADVANCED CANCER PAIN MANAGEMENT}

${ }^{1}$ Kate Marley, ${ }^{2}$ Manohar Sharma, ${ }^{2}$ Manish Gupta, Kathryn Wilford $2 .{ }^{1}$ Woodlands Hospice, Liverpool, UK; ${ }^{2}$ The Walton Centre, Liverpool, UK

10.1136/bmjspcare-2019-HUKNC.165

Woodlands Hospice hosts a weekly Joint Pain and Palliative Care clinic in collaboration with Aintree University Hospital and The Walton Centre. The service would qualify as a tertiary service as described by the Faculty of Pain Medicine's framework for cancer pain management (2019). The Walton Centre offers a range of interventions and is one of only three centres in Britain offering percutaneous cervical cordotomy and patients frequently attend from a distance for this procedure.

This audit was borne of a concern that we were unable to help some patients due to their short prognosis and lack of fitness for procedures or because they had died prior to assessment. It is a prospective audit of all referrals to the service and the outcome of the referrals over a six month period.

Out of 43 referrals, seven patients died or were too unwell to attend for assessment within days of the referral being received. Most patients who were able to attend were offered an appointment between one and four weeks from the referral. A further seven patients were assessed by phone as unsuitable for intervention due to short prognosis, poor performance status or their pain being too diffuse to be helped by an intervention and were spared a fruitless journey to clinic.

This audit highlights that referrals often come too late for the service to be of benefit and we must investigate the reasons for this, whether it be a lack of awareness of availability of procedures or reluctance on the part of patients to consider interventions. We plan to survey our local referrers and provide education about procedures and develop a pain MDT in the first instance and repeat the audit once these measures are in place in order to maximise the number of patients who may benefit from the service.

\section{P-144 REVOLUTIONISING FALLS PREVENTION IN THE HOSPICE SETTING}

Alison Stirton-Croft, Emma Imam. Isabel Hospice, Welwyn Garden City, UK

10.1136/bmjspcare-2019-HUKNC.166

Background Falls cost the NHS approximately $£ 1$.5 billion per year. Up to one in three over the age of 65 fall each year (Age UK, 2010). Increasing ageing population will continue to exacerbate this. It has been predicted (Chartered Society of Physiotherapy, 2015) that local investment to physiotherapy services could lead to 1283 fewer falls which can lead to approximately $£ 2,121,757$ cost savings for the locality. Collaborative working enabled two members of staff to be trained as Otago instructors. Otago, founded in New Zealand is an evidenced based programme looking at strength and balance exercises, which has shown to significantly reduce the risks of falls and injuries (Thomas, Mackintosh, Halbert, 2010).

Aims To deliver a 12-week Otago course for people with long term life limiting conditions, reduce the risk of falls, provide interactive exercises for balance and strengthening and gain peer support and improvements to mental health and wellbeing in the process.

Method A comprehensive supervised once a week programme plus home exercise programme was set up following the Otago principles. The patients received an informative theoretical session on falls prevention. Standardised objective measures including the timed up and go, sit to stand in 30 seconds, unsupported stand and tandem stand were taken before and after the 12 week course. Qualitative feedback was also gained through the local views on care questionnaire.

Results Ten patients were recruited and eight completed. Two patients had to stop the course due to various other commitments. $50 \%$ patients improved their sit to stands. $100 \%$ improved in the timed up and go. Tandem stand and unsupported stand was achieved by $100 \%$. The subjective feedback was all very positive.

Conclusion The Otago course had a positive impact on patients over 12 weeks. This will likely lead to a reduction in any falls and subsequent costs on healthcare provision and increase in quality of life.

\section{P-145 RISING UP - FALLS IMPROVEMENT}

Deborah Pegram, Tom Osborne, Jane Naismith. St Joseph's Hospice, London, UK

\subsection{6/bmjspcare-2019-HUKNC.167}

Background We were aware from reviewing incidents that we had a high number of falls and repeat falls in 2017. We had 107 falls from 64 patients. Clearly, we aim to have a harmfree environment but also want to maintain patients' independence. We have therefore embarked on an improvement journey to significantly reduce falls whilst maintaining a patient-enablement approach.

$\operatorname{Aim}(s)$

- Reduction in number of falls on the inpatient unit with no restriction on patient enablement and independence;

- Developing a learning culture around falls;

- Increased staff knowledge around their role in preventing falls;

- Education for patients and their families.

Methods We created a multidisciplinary falls group where everyone's ideas and contribution is respected. This group:

- Reviewed assessment process to reflect NICE guidelines;

- Reviewed equipment;

- Monitored trends;

- Developed and implemented a 'Safe use of bed rails' policy;

- Developed and implemented 1:1 observation policy;

- Falls Information leaflet produced for patients and relatives;

- Creation of Post Falls Protocol;

- Ran falls prevention and awareness days which included training.

- Reviewed each patient's outcome measure of performance (AKPS) and phase of illness.

Results Applying an all falls matter approach, with the combination of comprehensive guidelines, staff education (including Dementia/Namaste training) the purchase of new alarmed low beds, and patient and family education, we have been able to halve the number of falls in 2018 and dramatically reduce the number of repeat falls. The majority of falls occur in patients with an AKPS of $50 \%$ and have a deteriorating phase of illness. 
Conclusions Our approach has demonstrated a significant reduction in the number of falls without impacting on patients' independence. Quality of life and increased confidence in patients and staff is marked. We have seen a culture change within our team, where all falls and near miss falls are treated with the same importance.

\section{P-146 MUSIC TO MY EARS: IMPLEMENTING PERSONALISED MUSIC PLAYLISTS IN A HOSPICE INPATIENT UNIT (IPU)}

Kim Donaldson, Jen McLean, Liz Collins, Fiona Cruickshank, Erna Haraldsdottir. St Columba's Hospice, Edinburgh, UK

\subsection{6/bmjspcare-2019-HUKNC.168}

Background Creating individualised playlists for people living with a dementia has positive benefits including the reduction of behavioural and psychological symptoms (Bowell \& Bamford, 2018; Abraha, Rimland, Trotta, Dell'Aquila et al., 2017). Playlist for Life is a charity which is committed to making such playlists easily accessible for all and believes that music which is meaningful can help people with dementia to reconnect with those important to them. As hospices across the UK have been urged to build skills needed to support people with dementia (Hospice UK, 2015) we decided to implement Playlist for Life within our inpatient unit as a creative way of offering support to patients and their families.

Aims

- To enhance person-centred care offered to people with a dementia and their families;

- To use personalised playlists as a therapeutic tool to enrich connections between patients, families and staff.

Methods A practice development approach using collaboration, inclusion and participation (Manley, Parlour \& Yalden, 2013) was used throughout including:

- A dementia/cognitive impairment multi-professional group to oversee the project;

- Working with Playlist for Life to support the development of our project;

- Working with non-clinical departments to secure funding (Tulip Charitable Trust and J. Murray Napier Fund) and equipment;

- Reflections on practice with the Hospice Practice Development Facilitator and clinical staff;

- Written reflective accounts of all episodes of practice;

- 1:1 and small group sessions with clinical staff.

Results Although our initial aim was to work with people with a dementia, we have also worked with people with other forms of cognitive impairment and with no cognitive impairment. Using Playlist for Life has:

- Enabled life story telling and reminiscing;

- Supported the management of breathlessness and anxiety;

- Increased sense of wellbeing;

- Supported advance care planning;

- Been a legacy for family members.

Our next steps are to:

- Continue to establish an evidence base in a hospice setting through further evaluation

- Establish referral criteria.
Conclusion Individualised music playlists are an effective therapeutic tool in a hospice setting.

\section{P-147 LIGHTS PLEASE! CREATING MULTI-SENSORY SPACES IN AN ADULT HOSPICE}

Gemma Allen. The Mary Stevens Hospice, Stourbridge, UK

10.1136/bmjspcare-2019-HUKNC.169

Background Developing services to deliver personalised care for patients and visitors has been at the forefront of an indepth diversity and inclusion project. Lack of indoor sensory spaces was highlighted for those living with cognitive and visual impairments, anxiety, dementia and learning disabilities.

Aim Transform hospice space, providing patients and visitors with an area to relax, creating multi-sensory experiences adapted to a person's individual requirements.

Method Grant applications submitted to purchase specialist sensory equipment were successful with $£ 20,000$ received, primarily to aid, enhance and support health and wellbeing for more people in our hospice. The grants enabled the scoping and purchasing of specialist mobile equipment, rather than a static sensory room. Therefore, equipment is used across departments and accessible to all, including in-patient unit, day services, and family and bereavement support. Likewise, mobile equipment can be assembled in a specified room during holistic therapies or group activities.

Results The equipment consists of a deluxe sensory unit, containing bubble tubes, lights, aroma diffuser, music and projector. Additional mobile bubble tubes, lights, diffusers, music, projectors, VR headset, weighted duvet, sensory weighted chair and footstool and projector wheels were also purchased. The chair and footstool have received positive feedback from staff and patients.

'We have used the chair with a patient living with dementia. I had tried it myself and found it comforting but even so I was really surprised with how quick the calming effect was for our patient'

The chair stimulates senses, providing feelings of safety and security. Filled with balls the pressure increases tactile, muscle and joint sensations, sending impulses to the central nervous system resulting in improved feelings of wellbeing. We have identified an increased number of patients and visitors who will use this equipment whilst improving staff awareness about the therapeutic benefits of sensory spaces in adult hospices.

\section{P-148 DESIGNING AND DEVELOPING ON-LINE EDUCATION RESOURCES ABOUT SEXUALITY WITH CO-RESEARCHERS}

Maddie Blackburn, Sarah Earle, Lucy Watts. The Open University, Milton Keynes, UK

\subsection{6/bmjspcare-2019-HUKNC.170}

Background Inclusive research indicates that the decision-making process is one in which the 'professional' researcher and the 'co-researcher'(s) have equal weight and authority within the research process and collaborate throughout.

Aims In this presentation, we shall draw on our current project, focussing on the merits and some of the challenges in engaging co-researchers in inclusive research. This 